\title{
The Future of the Cytokine Discipline
}

\author{
Joost J. Oppenheim \\ Cellular Immunology, Cancer and Inflammation Program, National Cancer Institute, National Institutes \\ of Health, Frederick, Maryland 21702 \\ Correspondence: oppenhej@mail.nih.gov
}

The study of cytokines has evolved from the detection of functional activities present in tissue culture supernatants to the characterization of the three-dimensional molecular structures of the cytokines and their receptors. Investigators studying cytokines need to have specialized expertise in using cytokine assays, assessing their receptor interactions, signal transduction, gene activation, and biological effects, and in the therapeutic utilization of agonists and antagonists. Cytokinology can therefore be considered a discipline. In this article, I have considered studies leading to the identification of novel cytokines, potential producers of cytokine mimics such as viruses and the microbiome, and the complex interactions of the cytokine network with our vital functions. Our ever-increasing success in using cytokines and, in particular, cytokine inhibitors therapeutically suggest that cytokinology will eventually become an independent discipline.

have been invited to contribute an article on the future of cytokine studies presumably because I have spent more time than most in trying to advance our research into the future. My previous paper on the past, present, and future of cytokines did not cause any embarrassment, probably because it was read by few and ignored by all (Oppenheim 2001). Nevertheless, my crystal ball is cloudy and I have always continued to be surprised by developments that have circumvented, sideswiped, or completely ignored my predictions. The past, however, provides prologue to the future. Although I am much better at reviewing the past than in predicting the future, I will try to predict some of the miracles of tomorrow based on what has happened yesterday and today.

\section{DEFINITION}

We should begin by defining the scope of cytokine studies. The definition of cytokines can be broadly based on their function as intercellular signals (Oppenheim 2001). They can be either cell-surface associated or secreted proteins that interact with specific cell-surface receptors resulting in the mobilization and or modulation of target cells. Although I dislike use of the term "modulation," it is appropriate because cytokines can either have stimulating or suppressive effects on host cells. Although intercellular signals by molecular entities such as lipids, carbohydrates, DNA, or RNA are generally not considered cytokines, they can function as such when complexed by cytokines. Those intercel-

Editors: Warren J. Leonard and Robert D. Schreiber

Additional Perspectives on Cytokines available at www.cshperspectives.org

Copyright (C) 2018 Cold Spring Harbor Laboratory Press; all rights reserved; doi: 10.1101/cshperspect.a028498

Cite this article as Cold Spring Harb Perspect Biol 2018;10:a028498 


\section{J.J. Oppenheim}

lular signals that are produced by specialized cells in tissues or glands and are persistently released into the bloodstream are considered endocrine hormones and growth factors that act at a distance from their site of origin. They are the subject of endocrinologists. In contrast, cytokines usually, but not always, act locally over a short range in tissues either in a paracrine or even in an autocrine fashion. When cytokines are produced in abundance because of overwhelming infections, autoimmune reactions, or injuries, they can spill over into the serum and have global effects, such as fever and behavioral changes, and can have deleterious effects, such as "cytokine storm, cytokine release syndrome or macrophage activation syndrome" (Grom et al. 2016). This potentially lethal syndrome can be treated with anti-inflammatory reagents such as steroids, anti-interleukin (IL)6 , or anakinra, an antagonist of IL-1 (Schulert and Grom 2015).

\section{HISTORICAL BACKGROUND}

Over the past $70+$ years, the field of cytokine research has developed in a miraculous manner beginning with the initial observation in 1944 that "pyrexins" in inflammatory exudates caused the systemic response of fever (Menkin 1944). This led in 1953 to the identification of bacterial-derived endotoxin as the inducer of an endogenous pyrogen present in extracts of leukocytes (Bennett and Beeson 1953). At the same time, intercellular nerve growth factors were discovered (Levi-Montalcini and Hamburger 1953). This was followed in 1957 by the discovery of nonantibody cell-derived antiviral factors called interferons (IFNs) (Isaacs and Lindenmann 1957). The development of improved in vitro tissue culture techniques led immunologists to discover "blastogenic factors" in 1965 , which activated lymphocytes to proliferate (Kasakura and Lowenstein 1965). This was soon followed by the detection in tissue culture supernatants of macrophage inhibitory factor in 1966 (Bloom and Bennett 1966; David 1966) and a cytotoxic factor called lymphotoxin in 1967 (Ruddle and Waksman 1967). By 1978, about 100 different cytokine activities produced by a wide variety of cell types had been described (Waksman 1978). These biological activities were biochemically uncharacterized and ungraciously termed "lymphodrek" by yours truly (Oppenheim and Gery 1993). The cytokine field was so chaotic and deficient in molecular characterization that peer review committees stopped further funding of cytokine studies for over a decade. Fortuitously, miraculous progress by molecular biologists and biochemists rescued the cytokine field and made it possible to obtain pure recombinant preparations of cytokines by the 1980s. This led to an explosive expansion in the identification and molecular characterization of the hundreds of cytokines known to be involved in regulating our development, host defense, and repair processes. In addition, the structural elucidation of cytokine receptors led to the classification of cytokines into families based on the identities of their receptors (Oppenheim and Feldmann 2001).

\section{NOVEL CYTOKINES}

Of course it is easy to predict that even more cytokines will be discovered, especially because gene chip assays are revealing the novel targets and links of our ever-expanding cytokine network. One example of this is the identification of alarmins, a subset of danger-associated molecular patterns (DAMPs), as initiators of host defense. The alarmins are constitutively available endogenous molecules released from the granules, cytosol, or nucleus of cells by cell injury or other stimuli. In the extracellular milieu, they function as intercellular signals that mobilize cells by interacting with chemotactic receptors such as G-protein-coupled receptors (GiPCRs) and, in addition, activate cells by interacting with receptors such as Toll-like receptors (TLRs) (Nie et al. 2016). It has been very surprising that chromatin-binding proteins such as HMGB1, HMGN1, IL-33, and IL- $1 \alpha$ also have extracellular functions and are potent in inducing the production of proinflammatory cytokines. Other alarmins are antimicrobial peptides (AMPs), such as defensins and cathelicidins, which also have immune-enhancing host-defense functions. It is likely that more AMPs 
and chromatin binders will be identified to have alarmin functions. In addition, a number of thoroughly studied immunoenhancing moieties such as S100 A8/9, some heat shock proteins, and complement components also behave as alarmins and can be considered as such.

There are other intercellular signals that behave more like cytokines than hormones, which should be considered as important topics for studies by cytokinologists such as the neuropeptides. The fact that some cytokines have dramatic effects on peripheral and central nervous systems functions such as inducing fever, fatigue, and malaise undoubtedly involves the induction of a number of neuropeptide signals. Conversely, it is also quite reasonable to predict that neuropeptides can induce the production of some cytokines (Karin and Clevers 2016).

\section{CYTOKINE MIMICS}

I must also consider the possible effects of the microbiome on the cytokine network. It is clear that persistent microbial stimulation serves to optimize our immune responses and this undoubtedly results in the induction of cytokines. There is also considerable evidence that viruses produce many cytokine mimics to their own advantage. Whether bacteria also do this has not been as clearly documented, but one can predict that evolution has created some microorganisms that directly respond to cytokines or have endogenous genes that encode for cytokine mimics/antagonists (Elenkov 2000).

\section{CYTOKINES ASSAYS}

One of the major issues about cytokines is that no one can define what is a "normal" range for serum cytokines. While pharmaceutical companies may have such data on thousands of individuals, it may be impossible to define what "normal" represents because it is very likely that variables such as age, race, sex, and time of day of serum sampling may all impact the results. Nevertheless, threshold levels of cytokines will be identified that, when crossed, serve as a marker for disease. Thus, one can predict that more sensitive cytokine assays will eventually be included in normal blood tests.

\section{BIOLOGICAL EFFECTS OF CYTOKINES}

Despite heroic attempts to utilize computers to circumvent the need to sacrifice animals to solve biological problems, it is clear that cytokinology is an experimental science. Hypotheses need to be challenged and tested in the laboratory. Nevertheless, systems biology can play an important role in helping us understand the potentially voluminous results. A cytokine is a signal that never occurs alone, it is part of a complex milieu. Depending on the mix, we may repel an invader or self-destruct. The integration of cytokine signals requires a systems approach that cytokinologists must be equipped to study. Our current experimental tools can generate such an overwhelming amount of data that adequate analysis requires the application of systems biology.

There have been recent reports of patients having antibodies to cytokines, such as IFN- $\gamma$ or IL-6. If these antibodies are neutralizing, it could potentially impact the overall host immune response and might certainly contribute to the patients' resistance to infection (Kampmann et al. 2005). In fact, those patients with antiIFN- $\gamma$ antibodies had a major immunodeficiency condition. Thus, new assays will be needed to assess the presence of these endogenous antibodies and whether they neutralize the cytokine activity. Means of overcoming these autocytokine reactions need to be developed.

\section{CYTOKINE SIGNAL TRANSDUCTION}

An incredible amount of effort and expertise has been devoted to identifying the intracellular transduction pathways resulting in gene activation initiated by cytokine-receptor interactions. Although many cytokines share these signaling pathways, the fact that they interact with unique receptors on unique subsets of cells accounts for the specificity of cytokine functions. Consequently, targeting shared signal transducers for therapeutic purposes has been difficult because of the undesirably broad inhibitory effects. Despite this, there has been some notable com- 


\section{J.J. Oppenheim}

mercial success in developing inhibitors of some of the Janus kinase (JAK) signal transducers as anti-inflammatory therapeutics (O’Shea et al. 2015). It is a safe prediction that more targetable signal transducers and effective inhibitors will be identified. These can be antagonistic antibodies or inhibitors linked to aptamers targeted to cellsurface proteins (i.e., aptamers are small RNA or DNA molecules that bind specifically to target proteins). Aptamers might also be used to inhibit a cytokine activity by specifically binding and neutralizing their targets. Studies of the inhibitory effects or microRNAs (miRNAs) that bind cytokine messenger RNAs (mRNAs) may also yield a better means of inhibiting cytokine production with the goal of reducing inflammation.

\section{CYTOKINE MUTATIONS}

A number of primary genetic cytokine deficiencies have also been identified, such as $\gamma_{c}$ and IL-7 receptor deficiencies as causes of severe combined immunodeficiency (SCID). cKit gain-offunction mutations and IFN- $\gamma$ receptor mutations have a deleterious phenotype; CXCR4 deficiency has a phenotype, etc. With the development of better and easier methods for genetic engineering, perhaps manipulation of genes by CRISPR on other techniques will lead to therapeutic benefits in the future by the correction of mutations in cytokines or their receptors or even elimination of these genes in appropriate target cells.

\section{THERAPEUTIC CYTOKINE AGONISTS}

The course of cytokine research has led to some other surprising results. For example, many attempts have been made to utilize cytokines therapeutically with limited success. A number of proinflammatory cytokines such as tumor necrosis factor (TNF) and IL-1 have been thoroughly tested for their clinical antitumor effects with disastrous undesirable side effects such as fever and shaking chills, hypotension, and shock. Only the therapeutic use of IFNs to treat a number of diseases such as multiple sclerosis, virally induced chronic hepatitis, and malignant melanoma and leukemias has met with a modi- cum of success (Antonelli et al. 2015). This is despite the fact IFN has a number of undesirable and unpleasant side effects such as arthralgias, depressions, fatigue, muscle aches, and anorexia. These undesirable consequences of IFN's clinical use have been elucidated by an experimental attempt to treat adenovirus-induced upper respiratory infections (URIs) by administration of IFN- $\alpha$ in nose drops. This study actually showed that the IFNs administered without the adenovirus caused the nasal congestion and stuffiness, sneezing, and other symptoms of the URIs. These observations taught us that the toxic side effects of cytokines such as TNF, IFN, IL-12, and IL-1, rather than being a result of the infectious agent, were responsible for our disease symptoms. Attempts to use antagonists of these cytokines to reduce these symptoms backfired because the consequent reduction in host defense enabled the infectious agents to flourish and cause more severe damage. Fortunately, IFN is at present often being replaced by more effective therapies of liver disease and cancers.

It's tough to make predictions, especially about the future.-Yogi Berra

Research often results in unanticipated effects. For example, the CXCR4 blocker AMD3100 was developed as a human immunodeficiency virus (HIV) drug, but is now actually used to mobilize hematopoietic stem cells (HSCs) from bone marrow. Cytokines have also met with modest therapeutic success in the treatment of various cancers as immunoenhancing stimulants. Cytokines such as IL-2, IL-7, and IL-15 are used to grow and maintain lymphoid cells in vitro. In addition, cytokines and growth factors have been used to grow and differentiate stem cells and develop organoids. Perhaps in the future we will use cytokines to culture entire tissues and organs.

Many of the proinflammatory cytokines were evaluated for their adjuvant capacity, and granulocyte macrophage colony-stimulating factor (GM-CSF) was found to be the most potent in attracting dendritic cells to inflammatory tumor sites and thus in enhancing antitumor immunity (Jinushi et al. 2008). More recently, some alarmins that activate various TLRs have been shown to be even more potent in augment- 
ing adaptive antitumor immunity. Consequently, the role of these cytokine-like agonists may prove of therapeutic benefit, especially when utilized in an adjuvant setting. They can expand preexisting antitumor immunity, which has been shown to exist by the use of checkpoint inhibitors (Davar and Kirkwood 2016).

Another concern requiring a comment is that, although the pro- and anti-inflammatory effects of cytokines have been the predominant focus of study, the role of the anti-inflammatory cytokines such as transforming growth factor $\beta$ (TGF- $\beta$ ), IL-10, epidermal growth factor (EGF), vascular endothelial growth factor (VEGF), etc., in healing repair and fibrotic scarring, probably deserves more attention. After all, the aftermath of inflammation is the restoration of homeostasis. Future studies of those growth factors and cytokines that are engaged in this task may lead to therapeutic benefits.

\section{THERAPEUTIC CYTOKINE ANTAGONISTS}

A major surprise to cytokine experts was the serendipitous discovery that antagonistic antibodies to some of the proinflammatory cytokines could provide major benefits in reducing the inflammation associated with a number of autoimmune and autoinflammatory diseases. This came about based on futile attempts to use antagonistic anti-TNF to treat septic shock by Jan Vilcek at New York University in collaboration with investigators at a small company, Centocor (Vilcek 2016). In desperation, the antibodies were then provided to investigators as possible treatments of autoimmune diseases such as rheumatoid arthritis and Crohn's disease, resulting in amazing success (Feldmann and Maini 2010). Although this has not cured these diseases, it has provided considerable symptom relief to millions of patients. Similarly, neutralizing antibodies to IL-6, IL-12, IL-23, and IL-1 have benefited patients with selected autoimmune and autoinflammatory diseases. The therapeutic applications of cytokine antagonists have proven useful in diverse disciplines such as rheumatology, oncology, and genetic defects. Although the pharmaceutical industry dislikes developing antibodies or receptors that need to be injected, the many attempts to develop peptide or protein antagonists that can be administered in a pill form have failed. However, based on the obvious advantages of this means of administering drugs, I hope that more and better oral cytokine antagonists will be developed.

Another promising application of cytokine antagonists is in the realm of checkpoint inhibitors. A number of cytokines such as IL-10, IL-27, and TGF- $\beta$ have regulatory immunosuppressive effects. Although they are presumably intended to maintain homeostasis and to prevent autoimmune reactions, unfortunately, they also block antitumor responses. Attempts made in various animal tumor models to utilize antibodies or other antagonists of these cytokines have had modest antitumor effects. More research needs to be done to establish their utility. In particular, they will probably contribute to antitumor therapy in conjunction with cocktails of tumor-enhancing reagents and more conventional antitumor therapeutics such as surgery, radiation, and chemotherapy. The same probably holds for checkpoint inhibitors such as anti-PD1, antiCTLA4, and others that have impressive antitumor benefits as stand-alone therapeutics in a subset of patients, but are more effective when given in a cocktail with other therapeutics. Of course, one can argue that these cell markers are not really cytokines, but since they are shed and many cytokines have membrane-associated forms, I do not see any need to disown them.

This also raises the need for novel and rapid methods to identify more soluble cytokine receptors. Such receptors by binding cytokines could act as antagonists and decrease the availability of cytokines in the serum or alternatively might even enhance cytokine biological activity when complexed to their soluble receptors as has been reported for IL-6 and IL-15. It is also likely that "superkines" will be genetically engineered that have specific affinity for cytokine receptor chains, thus increasing their biological activity and specificity, as has recently been shown for IL-2 and its interaction with the IL-2 receptor $\beta$ chain. Similarly, I predict that new hybrid chemokines will be generated that attract and focus on specific immune cell subsets on a targeted tissue (e.g., $\mathrm{CD} 4^{+}$and $\mathrm{CD} 8^{+} \mathrm{T}$ cells 


\section{J.J. Oppenheim}

or $\mathrm{T}$ regulatory cells [Tregs]) and thus reduce systemic toxicity that could occur upon intravenous infusion of cytokines/chemokines.

\section{CONCLUSIONS}

The current fragmentation of the field of cytokines into subgroups that focus on studies of chemotaxis, TNF, and hematopoietic factors will probably be a temporary phase as indicated by the fusion of the IFN and cytokine societies. I foresee cytokinology becoming a more coherent field of study. Based on the ever-burgeoning therapeutic successes of cytokine agonists and antagonists, will cytokinology develop into an autonomous discipline such as endocrinology? Many diverse human conditions have been identified, resulting in primary or secondary cytokine deficiencies and cytokine overproduction. In agreement with the consequences of under- and overproduction of endocrine hormones, abnormalities in the production and effects of cytokines are of concern to a variety of medical subspecialties. In the case for immunology, a subspecialty for allergy and immunology has already been established. Thus, cytokinology may be considered a subsection of immunology or, like endocrinology, may eventually become entirely independent.

Currently, the clinical use of cytokines is largely based on direct infusion of the cytokines or anticytokine antibodies into the patients. In some cases, such as IL-12, this has resulted in serious toxicity or even death. However, in the future, there will be more targeted cytokine therapy such as cytokines in skin creams to promote tissue healing, cytokines directed toward specific organs or immune cell subsets to suppress an immune response in that organ, or cytokines directly targeted to tumors, an approach that has been under study for quite some time without much clinical success as yet.

I probably speak for my many cytokinologist colleagues in expressing our gratitude for having had the opportunity to contribute to the dynamic field of cytokine research because of the support of the National Institutes of Health and other funding agencies. As a result, cytokinology has developed into an immunological discipline.
Fortunately, immunologists from an initial restricted focus on the role of antibody specificity in adaptive immunity have been sufficiently flexible to expand their horizons to encompass the role of innate immunity and nonspecific cytokines. By analogy, I optimistically expect students of cytokines to expand their horizons and include studies of all intercellular host defense signals in their discipline. The cytokine network connects and interacts with lipid moieties: nucleotides, growth factors, neuropeptides, alarmins, hormones, and microbial reagents. Although as investigators we have to focus on our own research topic, we must be open to consideration of how cytokines interdigitate with the many other systems engaged in maintaining host integrity. Furthermore, as indicated in this article, we will never solve all of the problems, but we have to keep in mind the clinical utility of focusing our studies on the identification of the therapeutic benefit of cytokine agonists and antagonists with the goal of helping patients.

\section{ACKNOWLEDGMENTS}

I am grateful for the constructive critical discussion and modifications of this review by Drs. Howard Young and Scott Durum, and for the superb editorial assistance of Ms. Sharon Livingstone.

\section{REFERENCES}

Antonelli G, Scagnolari C, Moschella F, Proietti E. 2015. Twenty-five years of type I interferon-based treatment: A critical analysis of its therapeutic use. Cytokine Growth Factor Rev 26: 121-131.

Bennett IL Jr, Beeson PB. 1953. Studies on the pathogenesis of fever. II: Characterization of fever-producing substances from polymorphonuclear leukocytes and from the fluid of sterile exudates. J Exp Med 98: 493-508.

Bloom BR, Bennett B. 1966. Mechanism of a reaction in vitro associated with delayed-type hypersensitivity. Science 153: $80-82$.

Davar D, Kirkwood JM. 2016. Adjuvant therapy of melanoma. Cancer Treatment Res 167: 181-208.

David JR. 1966. Delayed hypersensitivity in vitro: Its mediation by cell-free substances formed by lymphoid cellantigen interaction. Proc Natl Acad Sci 56: 72-77.

Elenkov I. 2000. Neuroendocrine effects on immune system. In Endotext (ed. De Groot LJ, et al.). MDText.com, South Dartmouth, MA. 
Feldmann M, Maini RN. 2010. Anti-TNF therapy, from rationale to standard of care: What lessons has it taught us? J Immunol 185: 791-794.

Grom AA, Horne A, De Benedetti F. 2016. Macrophage activation syndrome in the era of biologic therapy. Nat Rev Rheumatol 12: 259-268.

Isaacs A, Lindenmann J. 1957. Virus interference. I: The interferon. Proc R Soc Ser B Biol Sci 147: 258-267.

Jinushi M, Hodi FS, Dranoff G. 2008. Enhancing the clinical activity of granulocyte-macrophage colony-stimulating factor-secreting tumor cell vaccines. Immunol Rev 222: 287-298.

Kampmann B, Hemingway C, Stephens A, Davidson R, Goodsall A, Anderson S, Nicol M, Scholvinck E, Relman D, Waddell S, et al. 2005. Acquired predisposition to mycobacterial disease due to autoantibodies to IFN- $\gamma$. J Clin Invest 115: 2480-2488.

Karin M, Clevers H. 2016. Reparative inflammation takes charge of tissue regeneration. Nature 529: 307-315.

Kasakura S, Lowenstein L. 1965. A factor stimulating DNA synthesis derived from the medium of leukocyte cultures. Nature 208: 794-795.

Levi-Montalcini R, Hamburger V. 1953. A diffusible agent of mouse sarcoma, producing hyperplasia of sympatheticganglia and hyperneurotization of viscera in the chick embryo. J Exp Zool 123: 233-287.
Menkin V. 1944. Chemical basis of fever. Science 100: 337 338.

Nie Y, Yang D, Oppenheim JJ. 2016. Alarmins and antitumor immunity. Clin Ther 38: 1042-1053.

Oppenheim JJ. 2001. Cytokines: Past, present, and future. Int J Hematol 74: 3-8.

Oppenheim JJ, Feldmann M. 2001. Introduction to the role of cytokines and cytokine production by cells in inflammation and immunity. In Cytokine reference, pp. 3-20. Academic, Orlando, Fl.

Oppenheim JJ, Gery I. 1993. From lymphodrek to interleukin 1 (IL-1). Immunol Today 14: 232-234.

O'Shea JJ, Schwartz DM, Villarino AV, Gadina M, McInnes IB, Laurence A. 2015. The JAK-STAT pathway: Impact on human disease and therapeutic intervention. Annu Rev Med 66: 311-328.

Ruddle NH, Waksman BH. 1967. Cytotoxic effect of lymphocyte-antigen interaction in delayed hypersensitivity. Science 157: 1060-1062.

Schulert GS, Grom AA. 2015. Pathogenesis of macrophage activation syndrome and potential for cytokine-directed therapies. Annu Rev Med 66: 145-159.

Vilcek J. 2016. Love and science, pp. 1-264. Seven Stories, New York.

Waksman BH. 1978. Modulation of immunity by soluble mediators. Pharmacol Ther 2: 623-672. 


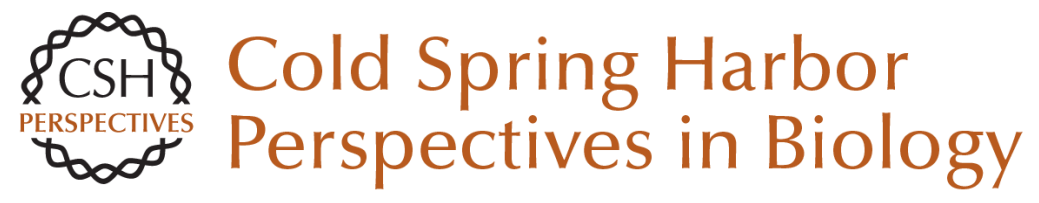

\section{The Future of the Cytokine Discipline}

Joost J. Oppenheim

Cold Spring Harb Perspect Biol 2018; doi: 10.1101/cshperspect.a028498 originally published online August 28, 2017

\section{Subject Collection Cytokines}

Interleukin (IL)-33 and the IL-1 Family of Cytokines -Regulators of Inflammation and Tissue Homeostasis

Ajithkumar Vasanthakumar and Axel Kallies

Targeting IL-10 Family Cytokines for the Treatment of Human Diseases Xiaoting Wang, Kit Wong, Wenjun Ouyang, et al.

Cytokine-Mediated Regulation of CD8 T-Cell Responses During Acute and Chronic Viral Infection

Masao Hashimoto, Se Jin Im, Koichi Araki, et al.

Cytokines in Cancer Immunotherapy

Thomas A. Waldmann

The Tumor Necrosis Factor Family: Family Conventions and Private Idiosyncrasies David Wallach

The Interferon (IFN) Class of Cytokines and the IFN Regulatory Factor (IRF) Transcription Factor Family

Hideo Negishi, Tadatsugu Taniguchi and Hideyuki Yanai
Interferon $\gamma$ and Its Important Roles in Promoting and Inhibiting Spontaneous and Therapeutic Cancer Immunity

Elise Alspach, Danielle M. Lussier and Robert D. Schreiber

Inflammasome-Dependent Cytokines at the Crossroads of Health and Autoinflammatory Disease

Hanne Van Gorp, Nina Van Opdenbosch and Mohamed Lamkanfi

Innate Lymphoid Cells (ILCs): Cytokine Hubs Regulating Immunity and Tissue Homeostasis Maho Nagasawa, Hergen Spits and Xavier Romero Ros

T Helper Cell Differentiation, Heterogeneity, and

Plasticity Jinfang Zhu

Development, Diversity, and Function of Dendritic Cells in Mouse and Human

David A. Anderson III, Kenneth M. Murphy and Carlos G. Briseño

Cytokines and Long Noncoding RNAs Susan Carpenter and Katherine A. Fitzgerald

For additional articles in this collection, see http://cshperspectives.cshlp.org/cgi/collection/

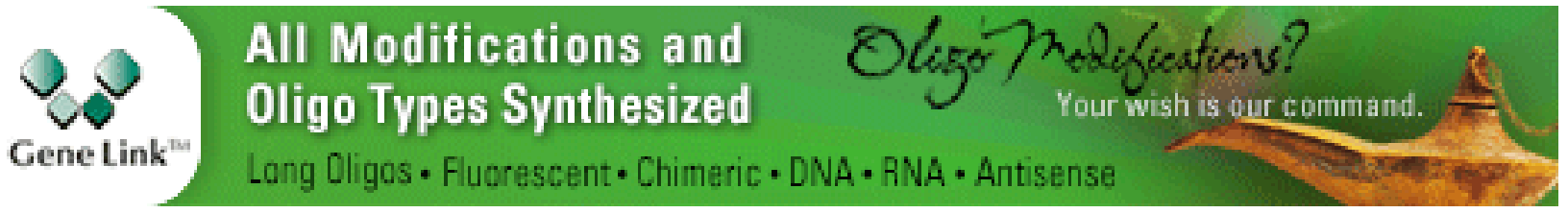

Copyright @ 2018 Cold Spring Harbor Laboratory Press; all rights reserved 
Role of the $\beta$ Common $(\beta \mathrm{c})$ Family of Cytokines in Health and Disease

Timothy R. Hercus, Winnie L. T. Kan, Sophie E. Broughton, et al.

Interleukin (IL)-12 and IL-23 and Their Conflicting Roles in Cancer Juming Yan, Mark J. Smyth and Michele W.L. Teng
Negative Regulation of Cytokine Signaling in Immunity

Akihiko Yoshimura, Minako Ito, Shunsuke Chikuma, et al.

Cancer Inflammation and Cytokines

Maria Rosaria Galdiero, Gianni Marone and Alberto Mantovani

For additional articles in this collection, see http://cshperspectives.cshlp.org/cgi/collection/

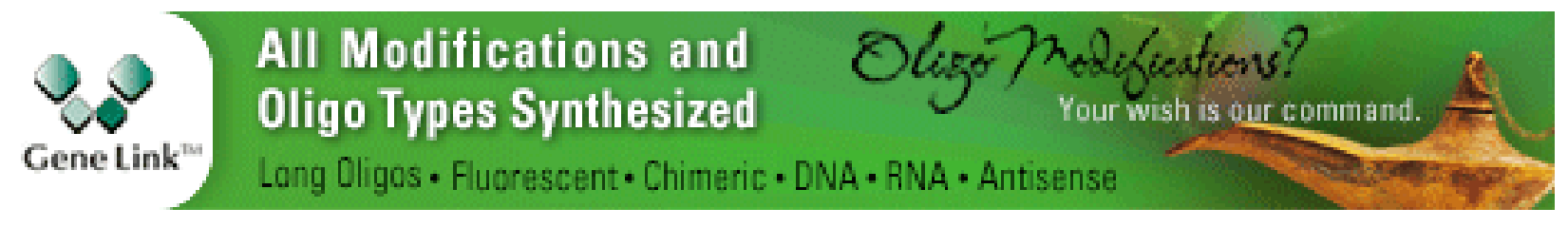

Copyright @ 2018 Cold Spring Harbor Laboratory Press; all rights reserved 